\title{
Controlled reversible debundling of single-walled carbon nanotubes by photo-switchable dendritic surfactants $\dagger$
}

\author{
Christian Kördel, ${ }^{a}$ Antonio Setaro, ${ }^{b}$ Pascal Bluemmel, ${ }^{b}$ Chris S. Popeney, ${ }^{a}$ Stephanie Reich ${ }^{b}$ and Rainer Haag ${ }^{* a}$
}

Received 8th February 2012, Accepted 21st March 2012

DOI: $10.1039 / \mathrm{c} 2 \mathrm{nr} 30305 \mathrm{a}$

\begin{abstract}
Stimulus responsive surfactants based on dendritic glycerol azobenzene conjugates were used to solubilize and debundle singlewalled carbon nanotubes in aqueous media. Their debundling property as well as their reaggregation behavior upon irradiation with light was examined and light triggered reversible bundling and precipitation are shown.
\end{abstract}

This paper describes for the first time the reversible switchable dissolution of single-walled carbon nanotubes (SWCNTs) in aqueous media. In general SWCNTs are poorly water soluble, which is a great drawback for their handling and application in biological systems for drug delivery, ${ }^{1}$ biosensors, ${ }^{2 a, b}$ ion channel blockers, ${ }^{3}$ biocatalysts ${ }^{4}$ and cellular uptake. ${ }^{5}$ The photoluminescence and photostability of SWCNTs, ${ }^{8}$ as well as their mechanical rigidity and high aspect ratio, which make them applicable as nanowires, ${ }^{7}$ were widely used in optoelectronic devices. Non-covalent functionalization of the nanotube surface has been commonly utilized to minimize the aggregation and promote dispersal of SWCNTs in aqueous solutions without perturbation of their electronic structure. ${ }^{6}$ Surfactants containing aromatic substrates such as pyrene ${ }^{8}$ or perylene, ${ }^{9}$ as well as aliphatic chains, are known to individualize and solubilize nanotubes in water. ${ }^{10,11}$

In common protocols sonication-centrifugation is used to disperse and separate CNTs in solution from impurities. However, under these harsh conditions a great loss of carbon nanotubes is inevitable. After the common dispersion with the sonication-centrifugation technique a new drying and redispersion would be necessary to allow the handling in further experiments requiring a new cycle of sonication-centrifugation with another loss of tubes. To overcome this problem a mild technique is needed to reversibly disperse CNTs in aqueous media that prevents the irretrievable waste of SWCNTs.

Recently, we described dendritic amphiphiles with the ability to switch their conformation due to the introduction of an azobenzene moiety between the alkyl chain and the glycerol dendron. ${ }^{12}$ These types of functional surfactants have the potential to release guest

${ }^{a}$ Freie Universität Berlin, Institut für Chemie und Biochemie, Takustraße 3 14195 Berlin, Germany. E-mail: haag@chemie.fu-berlin.de

${ }^{b}$ Freie Universität Berlin, Institut für Experimentalphysik, Arnimallee 14, 14195 Berlin, Germany

$\dagger$ Electronic supplementary information (ESI) available: Experimental methods and more details about the switching process. See DOI: $10.1039 / \mathrm{c} 2 \mathrm{nr} 30305 \mathrm{a}$ molecules because their critical micelle concentration increases dramatically with the light-induced trans-cis isomerization. Azobenzenes have been extensively used for molecular switches on surfaces. ${ }^{13 a-c}$ We introduced an azobenzene-glycerol conjugate with a pyrene anchor that was able to disperse CNTs, but the compound was not able to switch due to an overlap of the absorption spectra of pyrene and azobenzene. ${ }^{14}$

In this study switchable azo-based surfactants bearing an aliphatic chain and glycerol based dendrons were used to reversibly solubilize and debundle CNTs. As an amphiphile we choose an azobenzene with a third generation glycerol dendron and alkyl chain, subsequently referred to as $\mathrm{G} 3 \mathrm{azoC11}$ (Fig. 1). ${ }^{12}$ The solutions were prepared by the addition of HiPCO nanotubes to the aqueous surfactant solution followed by tip-sonication and centrifugation to yield high concentrations of debundled SWCNTs.

Initially, the G3azoC11 amphiphile was tested as a CNT debundling agent and, as the peaks in the luminescence map show, it was able to efficiently solubilize and debundle nanotubes in aqueous solution (Fig. 2a), as only individualized nanotubes display photoluminescence. Dendritic glycerol based amphiphiles showed an enhanced debundling efficiency with respect to SDS. ${ }^{11}$ The fluorescence peaks can be uniquely attributed to semiconducting SWCNTs of different chiralities $\left(n_{1}, n_{2}\right) \cdot{ }^{15}$ According to previous work the aromatic part between head and tail of the amphiphile has some influence on the selectivity of families of SWCNTs. ${ }^{16}$ To induce the trans to cis isomerization of the azobenzene moiety the sample suspension was irradiated at $366 \mathrm{~nm}$, resulting in a decrease of the

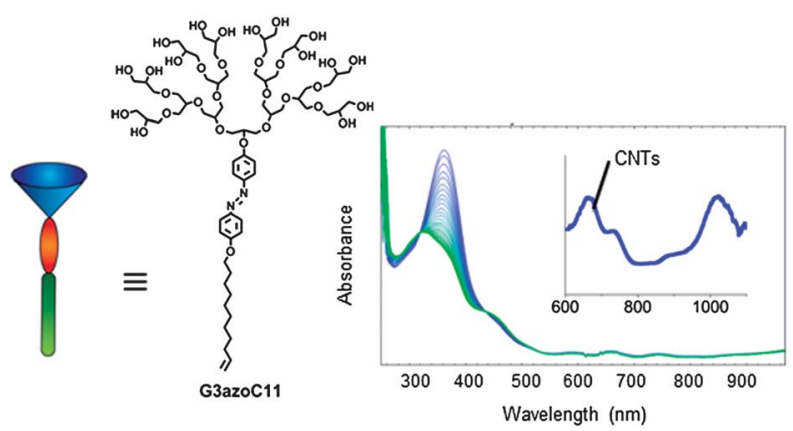

Fig. 1 Structure of the switchable amphiphile (left) and the corresponding UV/Vis spectrum of the isomerization process from a G3azoC11/SWCNT solution. 


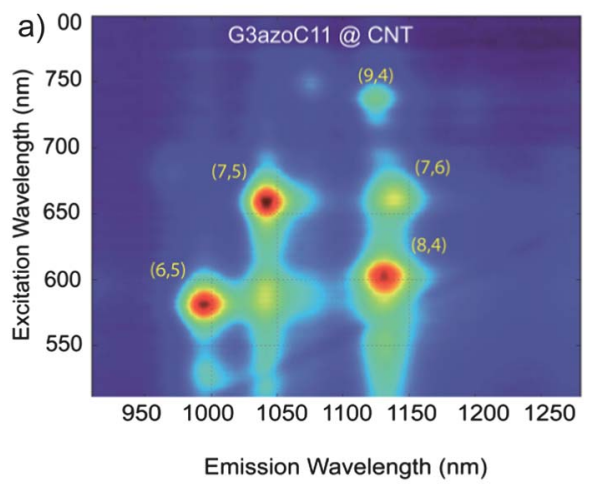

b)

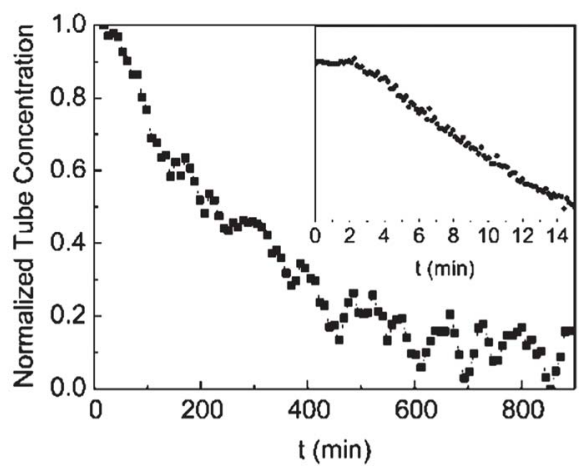

Fig. 2 (a) PLE map of a G3azoC11/CNT complex, demonstrating debundling of the tubes. (b) Change of the absorbance intensity of the $S_{22}$ transition at $670 \mathrm{~nm}$ of individual nanotubes during isomerization of G3azoC11 under $350 \mathrm{~nm}$ irradiation. The absorption intensity is proportional to the fraction of individual tubes in the solution. After a short time, the CNTs form bundles and precipitate in G3azoC11 solutions.

absorption maximum in the $350 \mathrm{~nm}$ region, which arises from the $\pi-$ $\pi^{*}$ transition of the trans isomer. At the same time the absorption increased at $450 \mathrm{~nm}$ from the $\mathrm{n}-\pi^{*}$ transition of the cis isomer (Fig. 1).

The aggregation behavior of the pure amphiphile G3azoC11 has been characterized previously. ${ }^{12}$ The critical micelle concentration (cmc) for the trans-isomer was $7 \times 10^{-6} \mathrm{M}$, whereas the $\mathrm{cmc}$ of the predominantly $c i$-isomer was 11-fold higher after the photo-isomerization. The supramolecular micellar structures can be distorted and destroyed by optical switching. The surfactant molecules are typically arranged like hemi-micelles or cylindrical micelles on the nanotube surface. ${ }^{17}$ The large geometrical change of the molecule is expected to destroy the supramolecular micellar structures. This is expected to induce a rebundling of the CNTs in solution.

The tube density in solution during the photo-induced switching of the dendritic glycerol azobenzene was examined. The absorbance at $670 \mathrm{~nm}$ is mainly caused by the absorbance band of the $S_{22}$ transition of mostly $(7,5)$ and $(7,6)$ nanotubes. ${ }^{15}$ During irradiation with UV light at $350 \mathrm{~nm}$ two periods were observed in the time dependence of the curve (Fig. 2b). During the first two minutes the absorbance is constant, followed by a steady decrease in absorbance. In the first two minutes the isomerization of the CNT-adsorbed azobenzene surfactant had no significant influence on the isolation and solubility of the nanotubes. The decrease in absorbance marks the point where CNTs started to aggregate and precipitate out of solution upon further irradiation. Herewith, the photo-isomerisation of the azobenzene moiety from the trans to the cis state affects the status of the tubes (Fig. 3).

In comparison to the pure azobenzene amphiphile solution, the switching process in switchable amphiphile/nanotube solution was slowed down. Measurement of the normalized intensities over time of the $\pi-\pi^{*}$ trans isomer adsorption indicated a difference in switching rates (Fig. 4). The pure surfactant solution reached the photostationary state in one minute, whereas the SWCNT-containing sample needed five times longer. The reversibility of the light-induced isomerization before nanotube precipitation was also verified (Fig. S1 $\dagger$ ) for five irradiation cycles. The retarded photoisomerization is believed to result from the isomerization of sterically hindered amphiphiles in an ensemble of surfactant molecules in hemi-micelles or cylindrical micelles on the nanotube surface. This is confirmed by our previous report on light induced micelle disruption of the pure
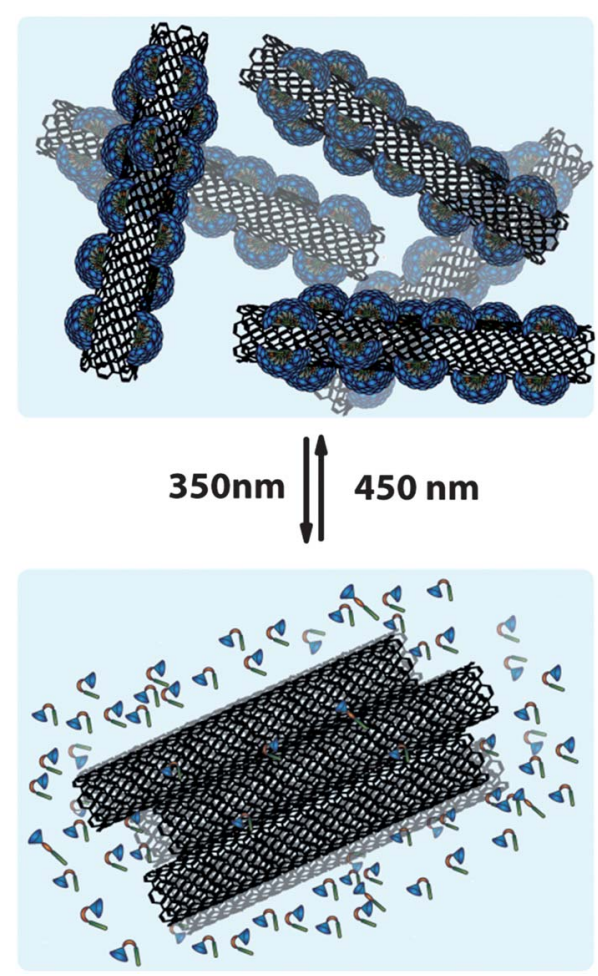

Fig. 3 Proposed mechanism of the reversible light-controlled solubilization and individualization of carbon nanotubes by dendritic G3azoC11 amphiphiles.

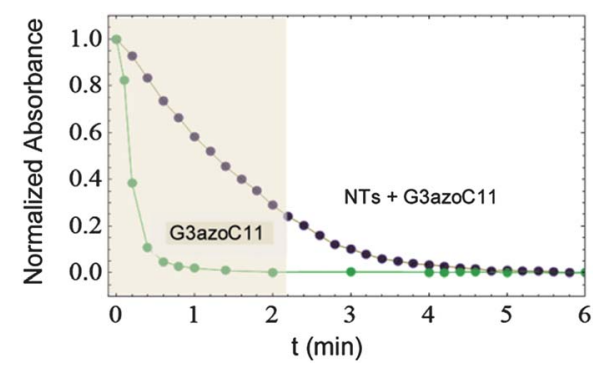

Fig. 4 Change in the absorbance intensity at $350 \mathrm{~nm}$ for pure surfactant solutions and the CNT/G3azoC11 suspension during irradiation. The grey zone indicates the region prior to the loss of solubilized CNTs. 
a)
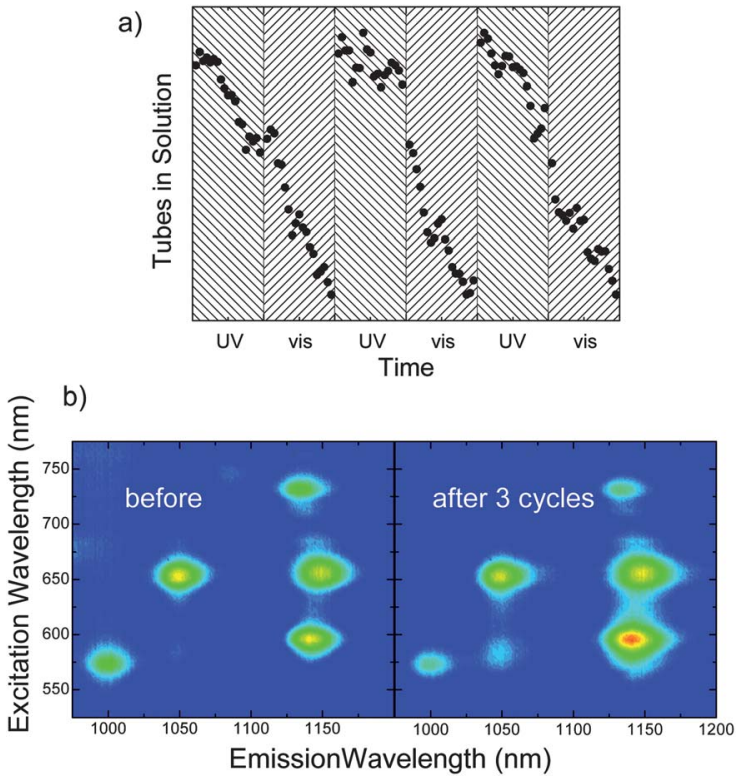

Fig. 5 (a) Single-walled carbon nanotube concentration derived from the background absorption@670 nm during three switching cycles. After each visible light irradiation a short sonication step was applied to stir up tube bundles. (b) PLE maps (same scale) before and after the three switching cycles, showing individualized tubes.

dendritic amphiphile G3azoC11. ${ }^{12}$ Further details of the switching dynamics are given in the $\mathrm{ESI} \dagger$.

So far, only the precipitation of individual tubes upon switching of the amphiphile was discussed. The reversibility of the bundlingdebundling step with the switchable molecules is demonstrated. The concentration of solubilized and debundled nanotubes was observed by UV/Vis spectroscopy upon three switching cycles (Fig. 5a). Switching from the trans to the cis isomer of the amphiphile with UV light led again to bundling and precipitation of the tubes, resulting in a decrease of the absorbance at $670 \mathrm{~nm}$. Immediate recovery of the amphiphilic properties by irradiation with visible light did not result in a higher amount of individualized nanotubes in solution, because they were precipitated and mostly sedimented on the bottom of the sample cuvette. Before the treatment with visible light a short sonication step is necessary to stir up these tubes. But in contrast to the initial and common preparation the sonication time was shorter (20 minutes instead of 60 minutes) and no ultracentrifugation was needed because all precipitated tubes were solubilized back. Upon cis-trans photoisomerization of the amphiphile, the former surfactant properties were regained. With this stir-up and back-switch treatment the nanotubes were resolubilized and debundled again, which is demonstrated by achieving the same absorbance intensity as before. This bundling-debundling process was repeated for three times without any loss of individualized tubes. Without back-switching of the amphiphile the nanotubes precipitate again. Additionally, the PLE map recorded after the three switching cycles proved the presence of individualized tubes (Fig. 5b). With this technique an easy handling of SWCNTs is now enabled without loss of the valuable material (Fig. 5a and b).

\section{Conclusions}

In summary we demonstrated a reversible solubilization and individualization of carbon nanotubes in an aqueous solution by a switchable surfactant. The trans-cis photoisomerization of the dendritic azobenzene amphiphiles triggered their detachment from the surface of the tubes. The nanotubes formed bundles as detected by the loss of their characteristic single tube absorption and luminescence. Switching the azobenzene in its original trans configuration resulted in a redispersion of the SWCNTs. The process was fully reversible for several circles. This stimulus-controlled supramolecular aggregate offers a way to switch the electronic and optical SWCNT properties as biosensors or in energy storage systems.

\section{Acknowledgements}

The authors thank the Deutsche Forschungsgemeinschaft (DFG) for financial support within the Sfb 658. Parts of the work were supported by the European Research Council (ERC) under grant number 210642.

\section{Notes and references}

1 M. Prato, K. Kostarelos and A. Bianco, Acc. Chem. Res., 2008, 41, 60.

2 (a) K. A. Williams, P. T. M. Vennhuizen, B. G. de la Torre, R. Eritja and C. Dekker, Nature, 2002, 420, 761; (b) C. J. Chen, S. Bangsaruntip, K. A. Drouvalakis, N. Wong Shi Kam, M. Shim, Y. Li, W. Kim, P. J. Utz and H. Dai, Proc. Natl. Acad. Sci. U. S. A., 2003, 100, 4984.

3 K. H. Park, M. Chhowalla, Z. Iqbal and F. Sesti, J. Biol. Chem., 2003, 278, 50212.

4 D. T. Mitchell, S. B. Lee, L. Trofin, N. Li, T. K. Nevanen, H. Söderlund and C. R. Martin, J. Am. Chem. Soc., 2002, 124, 11864.

5 X. Chen, G. S. Lee, A. Zettl and C. R. Bertozzi, Angew. Chem., Int. Ed., 2004, 43, 6111 .

6 M. J. O'Connell, S. M. Bachilo, C. B. Huffman, V. C. Moore, M. S. Strano, E. H. Haroz, K. L. Rialon, P. J. Boul, W. H. Noon, C. Kittrell, J. Ma, R. H. Hauge, R. B. Weismann and R. E. Smalley, Science, 2002, 297, 593.

7 S. J. Tans, A. R. M. Verschueren and C. Dekker, Nature, 1998, 672, 669.

8 X. Zhou, T. Zifer, B. M. Wong, K. L. Krafcik, F. Léonard and V. L. Vance, Nano Lett., 2009, 9, 1028.

9 C. Ehli, C. Oelsner, D. M. Guldi, A. Mateo-Alonso, M. Prato, C. Schmidt, C. Backes, C. F. Hauke and A. Hirsch, Nat. Chem., 2009, 1, 243

10 W. Wenseelers, I. I. Vlasov, E. Goovaerts, E. D. Obraztsova, A. S. Lobach and A. Bouwen, Adv. Funct. Mater., 2004, 14, 1105.

11 A. Setaro, C. S. Popeney, B. Trappann, V. Datsyuk, R. Haag and S. Reich, Chem. Phys. Lett., 2010, 493, 147.

12 C. Kördel, C. S. Popeney and R. Haag, Chem. Commun., 2011, 47, 6584.

13 (a) R. Klajn, J. F. Stoddart and B. A. Grzybowski, Chem. Soc. Rev., 2010, 39, 2203; (b) X. Zhang, Y. Wen, Y. Li, G. Li, S. Du, H. Guo, L. Yang, L. Jiang, H. Gao and Y. Song, J. Phys. Chem. C, 2008, 112, 8288; (c) S. Wagner, F. Leyssner, C. Kördel, S. Zarwell, R. Schmidt, M. Weinelt, K. Rück-Braun, M. Wolf and P. Tegeder, Phys. Chem. Chem. Phys., 2009, 11, 6242.

14 P. Bluemmel, A. Setaro, C. S. Popeney, R. Haag and S. Reich, Phys. Status Solidi B, 2010, 247, 2891.

15 S. M. Bachilo, M. S. Strano, C. Kittrell, R. H. Hauge, R. E. Smalley and R. B. Weisman, Science, 2002, 298, 2361.

16 A. Setaro, C. S. Popeney, B. Trappmann, R. Haag and S. Reich, Phys. Status Solidi B, 2010, 1.

17 E. J. Wallace and M. S. P. Sansom, Nanotechnology, 2009, 20, 045101. 